\title{
Triggering of drug relase of particles in hair follicles
}

Mak Wing Cheung, Patzelt Alexa, Richter Heike, Renneberg Reinhard, Lai Kwok Kei, Rühl Eckhardt, Sterry Wolfram and Lademann Jürgen

\section{Post Print}

N.B.: When citing this work, cite the original article.

Original Publication:

Mak Wing Cheung, Patzelt Alexa, Richter Heike, Renneberg Reinhard, Lai Kwok Kei, Rühl Eckhardt, Sterry Wolfram and Lademann Jürgen, Triggering of drug relase of particles in hair follicles, 2012, Journal of Controlled Release, (160), , 509-514.

http://dx.doi.org/10.1016/j.jconrel.2012.04.007

Copyright: Elsevier http://www.elsevier.com/

Postprint available at: Linköping University Electronic Press http://urn.kb.se/resolve?urn=urn:nbn:se:liu:diva-86351 


\section{Triggering of drug release of particles in hair follicles}

Wing Cheung Mak ${ }^{1}$ Alexa Patzelt ${ }^{2}$, Heike Richter ${ }^{2}$, Reinhardt Renneberg ${ }^{1}$, Kwok Kei $^{2}$ Lai $^{1}$, Eckhardt Rühl ${ }^{3}$, Wolfram Sterry ${ }^{2}$, Jürgen Lademann ${ }^{2, *}$

${ }^{1}$ Department of Chemistry, The Hong Kong University of Science and Technology, Clear Water Bay, Kowloon, Hong Kong, China

${ }^{2}$ Center of Experimental and Cutaneous Physiology (CCP), Department of

Dermatology, Venerology and Allergology, Charité - Universitätsmedizin Berlin, Charité - Universitätsmedizin Berlin, Germany

${ }^{3}$ Institute of Chemistry and Biochemistry, Freie Universität, Berlin, Takustr. 3, 14195

Berlin, Germany

Corresponding author:

*Prof. Dr. Jürgen Lademann

Charité - Universitätsmedizin Berlin

Department of Dermatology, Venerology and Allergology

Center of Experimental and

Applied Cutaneous Physiology

Charitéplatz 1

D-10117 Berlin

Telephone: ++4930450518100

Fax: $\quad$ ++4930450518918

Email: juergen.lademann@charite.de 


\section{Abstract}

Particulate drug delivery via hair follicles represents a promising concept, although requirements are high. This process must be realized at the desired depth and at the appropriate time, due to the fact that the particles themselves are not able to overcome the follicular skin barrier.

In the present study, a novel triggering concept for the release of a model drug from the delivering particles is presented basing on the application of two different particle types of the same size, where one particle type is the drug carrier, and the second one is loaded with a protease. The latter one is supposed to interact with the drugcarrying particles to trigger the drug release. A mixture of both particles was applied on porcine skin samples, followed by follicular analysis. As controls, the particles were applied unaided without protease, and one skin area remained untreated. The investigations revealed that the protease was able to release the model drug from the delivering particles in significant depths within the hair follicle (866 $\pm 62 \mathrm{~nm})$. Additionally, an uptake of the model drug in the sebaceous gland was observed after release providing a promising novel approach for the development of treatment strategies of different skin diseases.

Keywords: protein particles, transepidermal delivery, hair follicle, triggered release, skin 


\section{Introduction}

Hair follicles represent an interesting target structure for drug delivery, since they are surrounded by a dense network of blood capillaries [1-3] which is important for a rapid uptake of topically applied drugs. Additionally, dendritic cells [2] and stem cells [3], important for immunology and regenerative medicine, respectively, are closely associated with the hair follicles rendering them even more attractive for the development of selective drug delivery systems. Recently, it was demonstrated that particles in general [4-9] and especially at a size between 300 and $600 \mathrm{~nm}$ penetrate very efficiently and deeper into the hair follicles than non-particulate substances [4, 10]. Likewise, the storage time was found to be increased by up to 10 days, in the case of the particulate substances, which is significantly longer than 4 days, as observed for the non-particulate substances [11, 12].

As an explanation, Lademann et al. hypothesized that the surface structure of the hair and the hair follicle, as determined by the thickness of the keratin cells, which is in human hair of the order of $530 \mathrm{~nm}$ and in porcine hair of the order of $320 \mathrm{~nm}$, might act as a pumping system which pushes the particulate substances mechanically into the hair follicles when the hairs are in motion [13]. In contrast, in vitro, the movement of the hair has to be simulated by a massage appliance [11], under in vivo conditions, whereby a continuous movement of the hairs proceeds, pushing the particles efficiently and deeply into the hair follicles, if their size corresponds to the thickness of the keratin cells. Patzelt et al. [14] revealed that, depending on the size of the nanoparticles, different depths and thereby different target structures within the hair follicle can be reached.

However, the particles alone are not capable of reaching the target structures directly. Although, a transfollicular penetration of particles could be observed when the skin barrier was strongly disrupted [9], several other studies demonstrated that particles of this dimension do not pass the intact barrier of the hair follicles $[5,15,16]$, Therefore, loading of the particles with a drug represents an efficient opportunity to transport the drug into the hair follicles provided that, after reaching the specific depth within the hair follicles, the drug is released from the particles to penetrate independently through the intact barrier of the hair follicles, as shown previously [17]. A main advantage of this type of carrier system is the increased efficacy, as larger quantities of drugs can be administered to the contemplated target sites when transported by particles [18, 19]. Applying this carrier concept, it is essential to 
quickly release the drug from the particles onto the specified target structure, at the appropriate time [17]. The use of porous particles, continuously releasing the drug, seems to be less appropriate, since the release would already occur in the storage container before skin application. The application of defined amounts of drugs would thus be prevented. Recently, it could be demonstrated that the release of a model drug (fluorescent dye) from particles composed of bovine serum albumin (BSA) based on protein activation and spontaneous self-assembly technique at a size of $500 \mathrm{~nm}$ can be decomposed by an interaction of the protease [17]. In these experiments, the drug-containing particles were applied onto the skin before the protease contained in a liquid formulation was applied. The release procedure of the model drug from the particles was analyzed by the time dependent fluorescence properties of the dye, as described by Mak et al. [17]. It was found that the particles penetrated down to the sebaceous gland of the hair follicles, whilst the liquid protease penetrated only into the upper third part of the hair follicles. This behavior is typical for non-particulate substances. Consequently, drug release occurred only in the upper third of the hair follicles, whilst the deeper target structures were not able to interact with the drug.

Therefore, a novel concept to trigger the release of a drug from the particles inside the hair follicles is presented in the current study. It is based on the application of two different types of particles of the same size, supposed to penetrate efficiently into the hair follicles. One type of particle is the drug carrier, whereas the second particle type interacts with the drug-carrying particles and triggers the drug release. The drug release from the particles is investigated by changes in fluorescence properties of the model drug, as described by Mak et al. [17]. The concept of the penetration and release process is schematically depicted in Figure 1. 


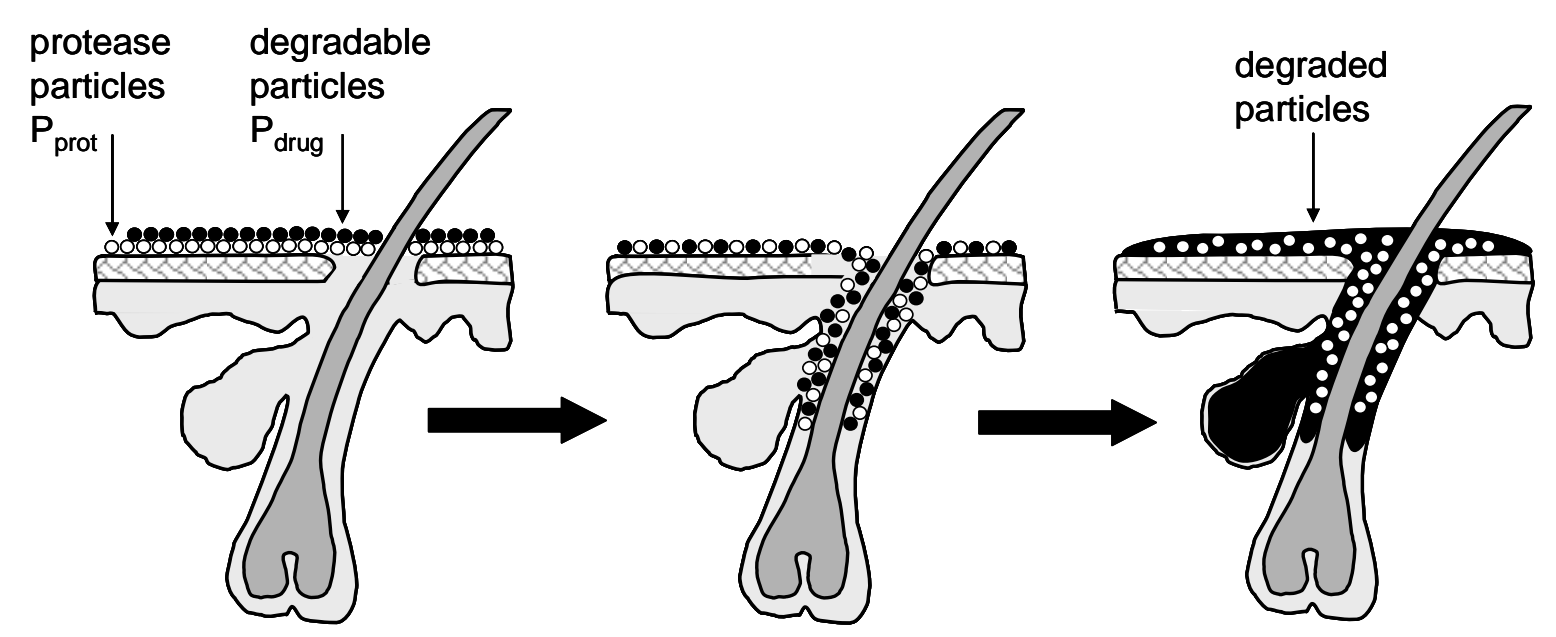

Figure 1. Schematic diagram of penetration and release concept

\section{Materials and methods}

\section{Materials}

Calcium chloride and sodium carbonate were purchased from Sigma-Aldrich (Missouri, USA). Bovine serum albumin (BSA), dithiothreitol (DTT), fluorescein isothiocyanate (FITC), and protease from streptomyces griseus were obtained likewise from Sigma-Aldrich (St. Louis MO, USA). Ethylenediaminetetracetic acid (EDTA) was purchased from Invitrogen (California, USA).

\section{Particle preparation}

Two types of particles were used in this study. The first type consisted of BSA particles loaded with FITC (model drug) and had a mean diameter of $532 \pm 24 \mathrm{~nm}$ $\left(P_{\text {drug }}\right)$. The fabrication of $P_{\text {drug }}$ is based on co-precipitation of BSA molecules and FITC molecules within $\mathrm{CaCO}_{3}$ templates, followed by DTT induced crosslink of BSA molecules via disulfide bridges to form BSA particles loaded with FITC. Finally, the $\mathrm{CaCO}_{3}$ templates were removed $[20,21]$. In brief, a solution containing calcium chloride $(0.5 \mathrm{M})$, BSA $\left(10 \mathrm{mg} \mathrm{mL}^{-1}\right)$, and FITC $\left(1 \mu \mathrm{g} \mathrm{mL}^{-1}\right)$ was rapidly mixed with sodium carbonate $(0.5 \mathrm{M})$ and was subsequently stirred for 30 seconds to produce BSA and FITC loaded $\mathrm{CaCO}_{3}$ particles. The BSA and FITC loaded $\mathrm{CaCO}_{3}$ particles were washed five times by centrifugation (2000 rpm, 2 minutes, Eppendorf centrifuge 5415D, Germany) and re-dispersion cycles. The resulting BSA and FITC loaded $\mathrm{CaCO}_{3}$ particles were incubated with $1 \mathrm{mM}$ DTT solution at $\mathrm{pH} 7.5$ for 15 minutes. Subsequently, the $\mathrm{BSA}$ and FITC loaded $\mathrm{CaCO}_{3}$ particles were washed five times to 
remove the DTT, re-suspended in a buffer solution $(\mathrm{pH} 7.5)$, and incubated for 1 hour. Finally, the $\mathrm{CaCO}_{3}$ template was removed by addition of EDTA $(0.2 \mathrm{M})$ and incubated for 1 hour at room temperature. The resulting $P_{\text {drug }}$ was washed twice by centrifugation (2000 rpm, 2 minutes) and re-dispersed in 1X phosphate buffer saline (PBS) at $\mathrm{pH} 7.4$.

The second type of particles consisted of $\mathrm{CaCO}_{3}$ particles loaded with protease tagged with rhodamine $\left(\mathrm{P}_{\text {prot }}\right)$. With a mean diameter of $516 \pm 37 \mathrm{~nm}$, the size of $P_{\text {prot }}$ was similar to that of the drug loaded particles $\underline{P}_{\text {drug. }}$. In brief, a solution containing protease $\left(1 \mathrm{mg} \mathrm{mL}^{-1}\right)$ and calcium chloride $(0.5 \mathrm{M})$ was rapidly mixed with sodium carbonate $(0.5 \mathrm{M})$ and continuously stirred for 30 seconds to produce $P_{\text {prot. }}$. The $P_{\text {prot }}$ was washed five times by centrifugation (2000 rpm, 2 minutes) and re-dispersed in $1 \mathrm{X}$ phosphate buffer saline (PBS) at $\mathrm{pH}$ 7.4. By means of this approach, the amount of protease molecules incorporated inside the $\mathrm{CaCO}_{3}$ particles was determined by the physical interaction of the protease $/ \mathrm{CaCO}_{3}$ mixture during co-precipitation. Thus, protease molecules were slowly released from the $\mathrm{CaCO}_{3}$ during storage until equilibrium had been reached between the encapsulated and the bulk phase protease, which was, however, not the aim of our study.

The particles applied in this study have a model function, in order to demonstrate the efficacy of the penetration of the particles and the effect of triggered drug release, close to the target structures.

These two types of particles are demonstrated to be biocompatible and of high cytocompatibility being also applicable to humans.

\section{Tissue samples}

As this work required a high number of biopsies, the investigations were performed in vitro on porcine ear skin due to ethical reasons. Porcine ear skin provides a structure similar to human skin $[22,23]$ and is well suited for the investigation of the penetration of particles into the hair follicles, as previously shown [4, 10, 14]. Excised human skin is not applicable for follicular penetration studies, since human skin contracts directly after excision which leads to a permanent occlusion of the hair follicles by the contracted elastic fibers [24]. On the contrary, porcine ear skin remains fixed to the cartilage during investigation and therefore the follicles remain open [25]. The pig ears had been freshly obtained from the butcher and the 
investigations were completed within six hours. Prior to treatment, the pig ears had been rinsed under cold water and dried with paper towels. Approval for the experiments had been obtained from the Veterinary Board, District of Berlin-TreptowKöpenick.

\section{Application protocol}

Three skin areas of $4 \times 4 \mathrm{~cm}^{2}$ were marked on each porcine ear skin sample. Both types of particles, $P_{\text {drug }}$ and $P_{\text {prot }}$, were stored separately and were mixed directly prior to application at a relation 1:1. The mixture was then applied to the porcine skin on the first marked skin area (application protocol 1). On the neighboring skin area, pure $P_{\text {drug }}$ was exclusively applied (application protocol 2), the third skin area remained untreated as a control. In both cases the applied concentration was 2 $\mathrm{mL} / \mathrm{cm}^{2}$. The penetration of the particles into the skin was stimulated by a massage appliance for one minute, by means of the massage applicator (PC60, petra-electric, Burgau, Germany) in order to stimulate the movement of the hairs, similar to the in vivo situation.

\section{Preparation of skin samples}

Sixty minutes after application of the particles, circular biopsies of $3 \mathrm{~mm}$ diameter were removed from all skin areas treated with application protocol 1 or 2 and from the control area, respectively. The biopsies were shock frozen in liquid nitrogen and $10 \mu \mathrm{m}$ cryo sections were prepared. The study was performed on 6 pig ears; for each tissue sample, at least 10 hair follicles were analyzed.

\section{$\underline{\text { Sample analysis }}$}

The distribution of the particles in the cryo sections was determined by laser scanning microscopy in the transmission and fluorescent modes, utilizing the laser scanning microscope, LSM 2000 (Carl Zeiss, Jena, Germany) [17]. In the case of application protocol 1, when $P_{\text {drug }}$ and $P_{\text {prot }}$ were applied together, the fluorescent dyes, FITC and rhodamine, were excited at $488 \mathrm{~nm}$ by argon laser radiation, the fluorescence signal of FITC was detected at $520 \mathrm{~nm}$ and that of rhodamine at 590 $\mathrm{nm}$. In the case of application protocol 2 (application of pure $P_{\text {drug }}$ ), only the fluorescence signal of FITC was detected. 
In preliminary experiments, it was demonstrated that the fluorescence signal of the model drug FITC increased strongly, when $P_{\text {drug }}$ was dissolved after contact with Prot.

\section{Electron scanning microscopy}

The morphology of the particles was examined by scanning electron microscopy JSM-6700F (JEOL, Japan). Imaging samples were prepared by dropping the particle solution onto a flat silicon surface, air dried at room temperature and followed by gold deposition.

\section{Statistic analysis}

The penetration depths of the model drug FITC and the rhodamine-labelled protease particles were determined for at least 10 hair follicles per skin sample. For each skin sample, mean values and standard deviations were calculated with the software program Excel ${ }^{\circledR}$ (Microsoft, Washington, USA). The mean values were utilized for statistical evaluation. Therefore, the non-parametric Wilcoxon-test was used to compare the different depths using the software program SPSS ${ }^{\circledR}$ (IBM Cooperation, New York, USA). The significance level was set at $p<0.05$.

\section{Results}

Typical images of the $\mathrm{P}_{\text {drug }}$ and $\mathrm{P}_{\text {prot }}$ obtained from electron scanning microscopy are shown in Fig. 2. Both types of particles had a uniform spherical morphology with a similar average diameter of approximately $520 \mathrm{~nm}\left(532 \pm 24 \mathrm{~nm}\right.$ for $P_{\text {drug }}$ and $516 \pm$ $37 \mathrm{~nm}$ for $\mathrm{P}_{\text {prot }}$ ). The surface of the BSA particles (Fig. 2a) appeared smooth and less porous, whereas the $\mathrm{CaCO}_{3}$ particles showed seed-like agglomerates with a rough surface and large pores (Fig. 2b). The difference in particle morphology explains why the model drug (FITC) was encapsulated and strongly linked to $P_{\text {drug, whereas }}$ protease slowly diffused out of $P_{\text {prot. }}$. The diffusion process started when $P_{\text {prot }}$ was applied onto the skin, as this resulted in a strong change in the concentration gradient of the protease from the particles to the environment (skin). It is essential that free protease can interact with $\mathrm{P}_{\text {drug }}$ and cause decomposition of the particles, which releases the model drug inside the hair follicles. 


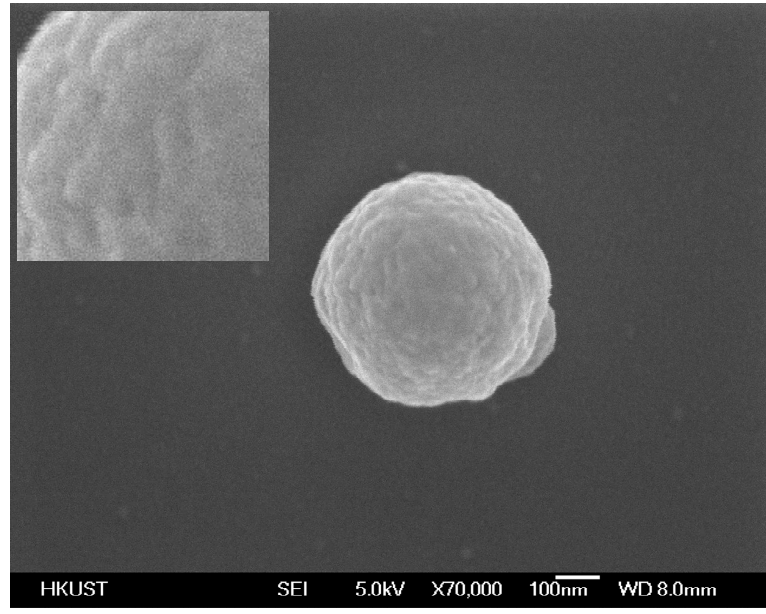

a

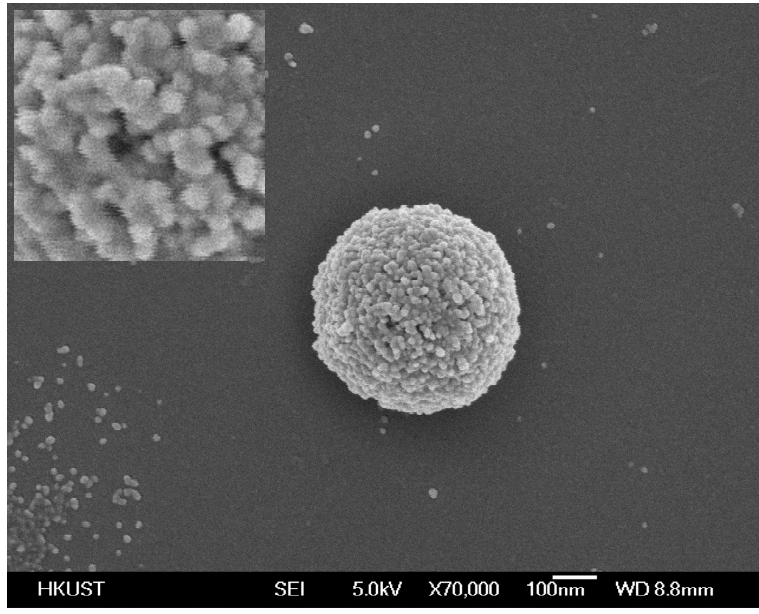

b

Figure 2. SEM images showing the surface morphologies of the $P_{\text {drug }}(a)$ and $P_{\text {prot }}$ particles (b). The top inset shows the enlarged image

The intensity of the fluorescence signal, resulting from the model drug FITC detected in the histological sections, was approximately one order of magnitude higher than with $P_{\text {drug }}$ when $P_{\text {drug }}$ and $P_{\text {prot }}$ were applied as a mixture (Fig. 3a), which was applied unaided (Fig 3b). We note that no fluorescent signal could be detected for the control skin sites, and identical results were obtained for all hair follicles in all tissue samples.

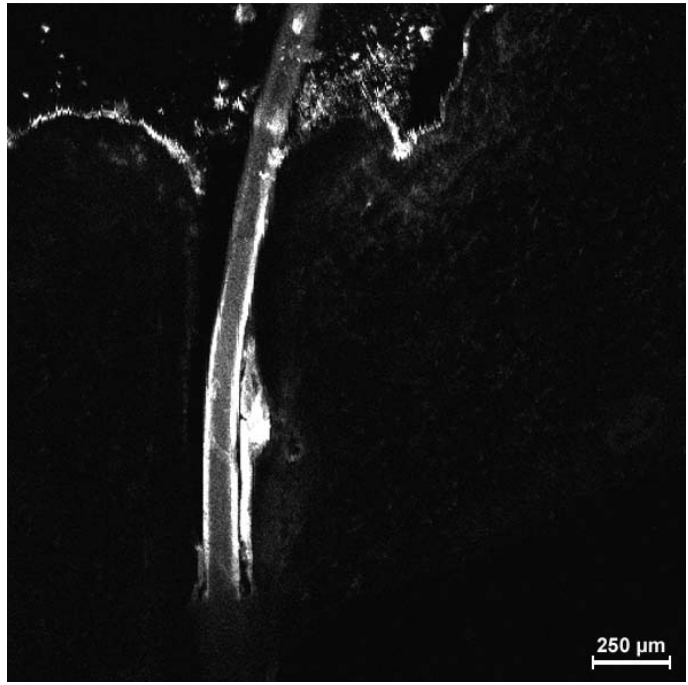

a

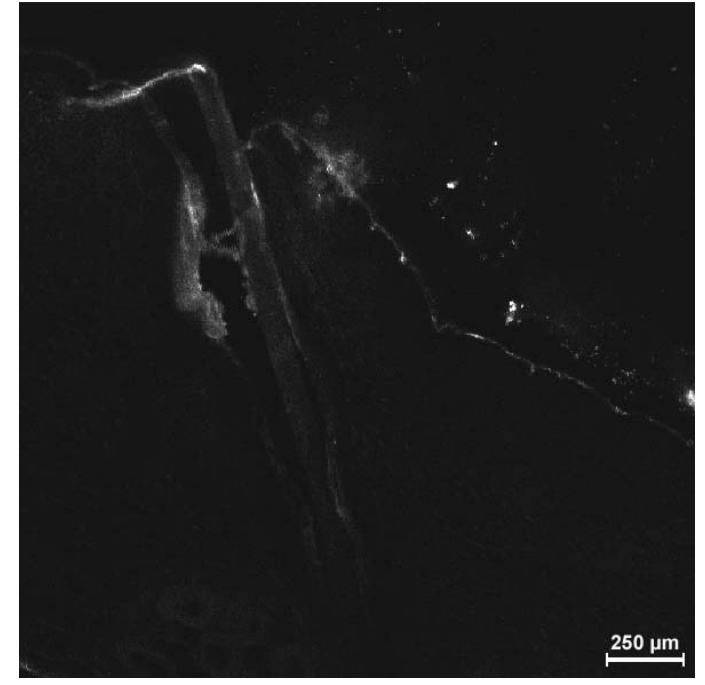

b

Figure 3. Differences in the fluorescence intensity of the model drug FITC when $\mathrm{P}_{\text {drug }}$ and $\mathrm{P}_{\text {prot }}$ were applied in a mixture $(\mathrm{a})$ or when $\mathrm{P}_{\text {drug }}$, was applied 
Fig. 4 shows the average penetration depths of the $P_{\text {drug }}$ and $P_{\text {prot }}$ particles applied as a mixture (application protocol 1). In all investigated hair follicles, a strong fluorescence signal was observed at $520 \mathrm{~nm}$ demonstrating that the model drug was released from the $P_{\text {drug }}$ as a result of the interaction of both types of particles causing the release of the drug. The fluorescence signal of rhodamine (protease particles) at $590 \mathrm{~nm}$ was detected at similar penetration depths $(p>0.05)$.

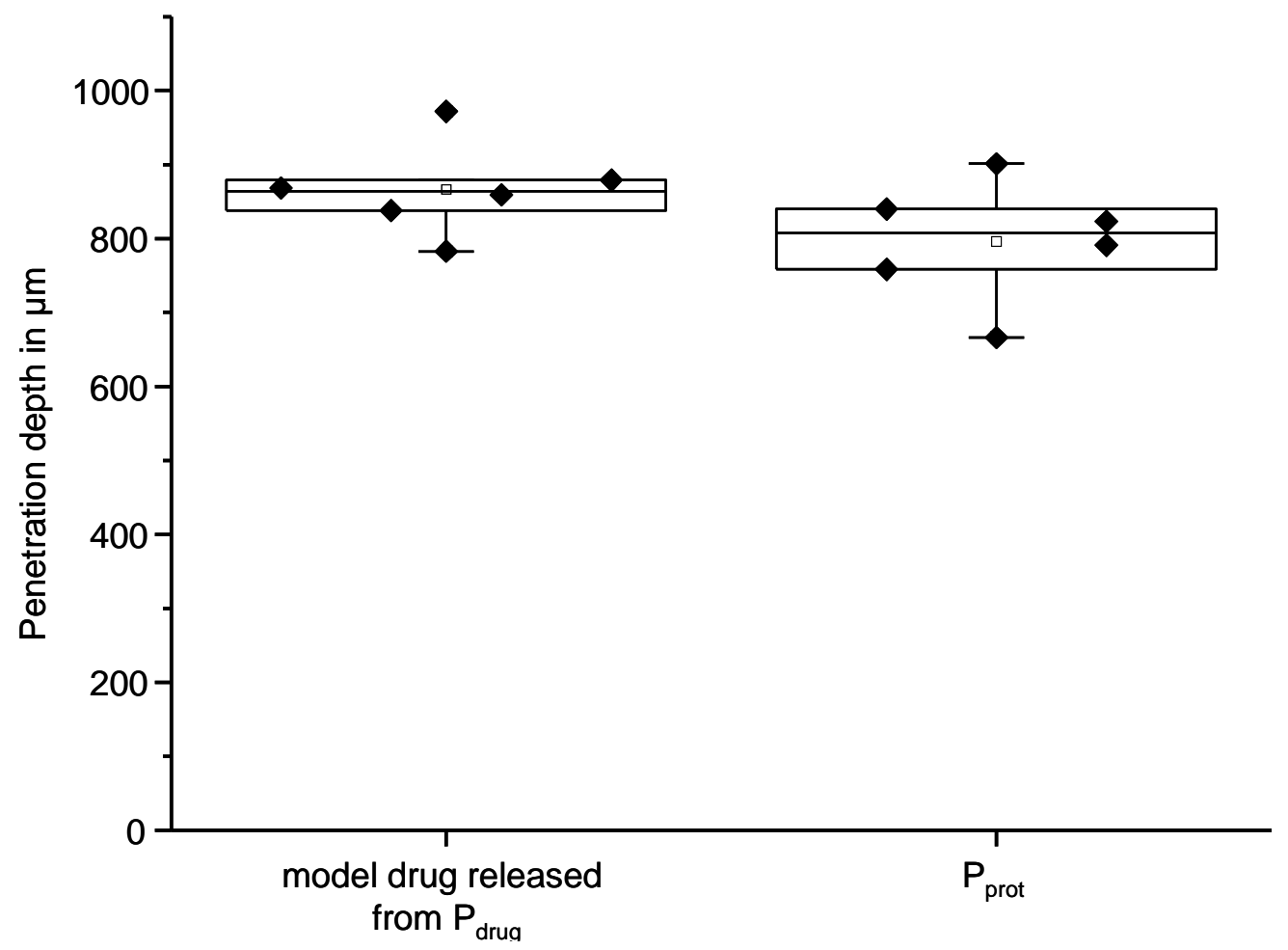

Figure 4. Average values of the penetration depths of the model drug FITC and the rhodamine-labelled protease particles in $\mu \mathrm{m}$

Additionally, in the case of application protocol 1, a strong fluorescence signal of fluoresceine was also detected in the sebaceous glands (Fig. 5a), whereas, when only $P_{\text {drug }}$ was applied unaided, not even a weak fluorescence signal could be observed in the region of the sebaceous glands (Fig. 5b). 


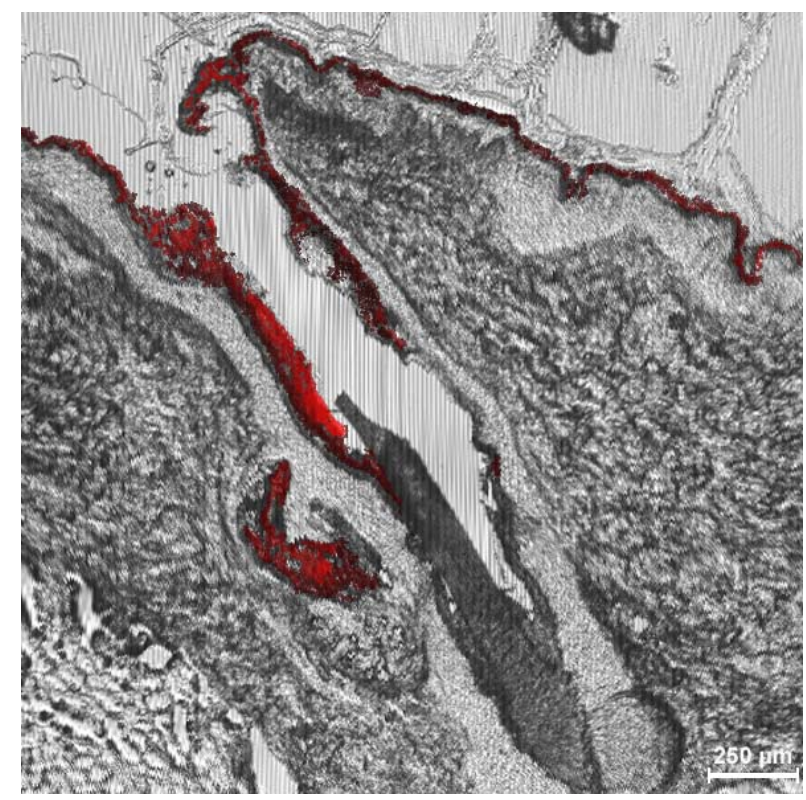

a

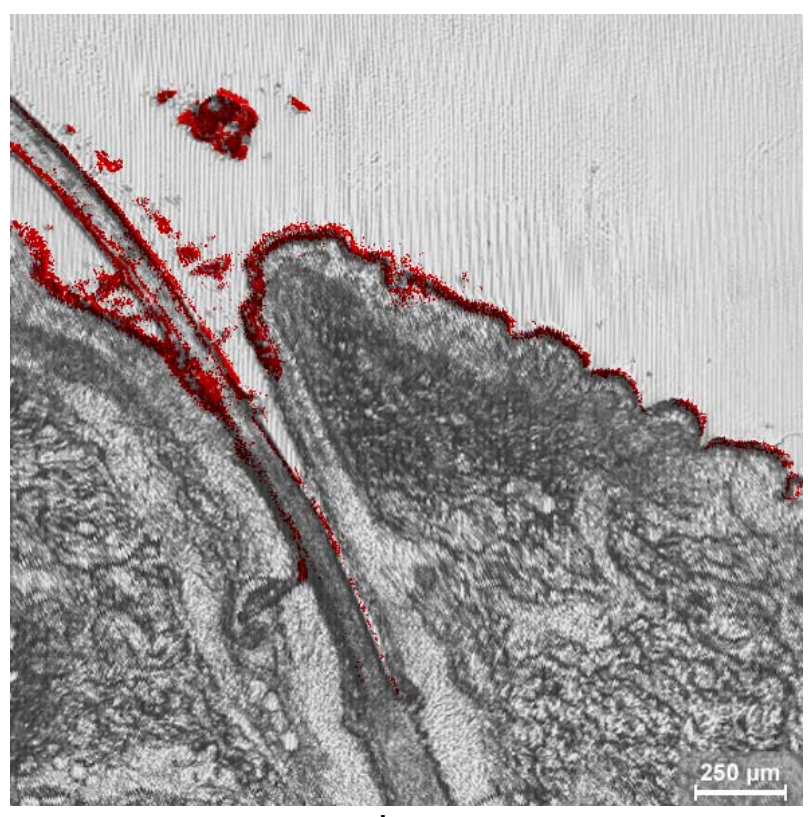

b

Figure 5. Distribution of the model drug FITC in the hair follicle and the sebaceous gland when $\mathrm{P}_{\text {drug }}$ and $\mathrm{P}_{\text {prot }}$ were applied as a mixture (a) or when $\mathrm{P}_{\text {drug }}$ was applied unaided without protease particles (b)

\section{Discussion}

Particle sizes between $300 \mathrm{~nm}$ and $600 \mathrm{~nm}$ penetrate more efficiently and deeper into the hair follicles than non-particulate substances [10]. However, particles in this size range are not able to overcome the intact skin barrier, neither the barrier of the stratum corneum nor the barrier of the hair follicles [5]. Efficient drug delivery through the skin barrier, however, can be obtained, if the particles are employed as carrier systems loaded with the drug of interest. In this case, the particle can deliver the drug close to the target structure of interest in the hair follicles where the drug is released and passes independently the skin barrier of the hair follicle. There is evidence that molecules, being transported into the hair follicles, are able to pass the barrier of the hair follicles and thus reach the living cells. This was shown, e.g., for the penetration of caffeine [26] and minoxidil [27]. In these experiments, the hair follicles were artificially closed, enabling a clear differentiation between follicular and intercellular penetration [28]. These studies revealed a faster penetration via the follicular pathway than the intercellular penetration pathway. Pharmacokinetic calculations revealed the absorption rate constant of caffeine for hair follicles to be nearly 10 
times higher than that for the stratum corneum and the fraction of absorption from hair follicles was more than half of that of the stratum corneum [29].

Particulate drug delivery via hair follicles, thus, represents a promising concept although the requirements are high, as the release of the active drug close to the target site of interest has to be realized at the desired depth and at the appropriate time. The penetration depths of the particles can be easily controlled by the particle size. Therefore, the drug release needs to be stimulated by a suitable trigger process.

In the present study, an advanced delivery system based on the interaction of drug loaded particles $\left(P_{\text {drug }}\right)$ with the triggering reagent protease, also being applied via particle formulation $\left(P_{\text {prot }}\right)$, was used to trigger the drug release inside the hair follicles. In contrast to the previous study reported by Mak et al. [17], where the protease was applied in form of a solution together with the drug loaded particles, a mixed particle formulation composed of $P_{\text {drug }}$ and $P_{\text {prot }}$ was applied for delivery and triggered release of drugs within the hair follicles close to the corresponding target structure. As $P_{\text {drug }}$ and $P_{\text {prot }}$ were of similar size, the penetration depths of both types of particles into the hair follicles were almost identical. A slight but not significantly increased penetration depth for the model drug FITC can be explained by a further independent penetration of the model drug after release. After topical application, a strong change in the concentration gradient for protease occurs, which induces protease to diffuse from the particle carriers, after which the protease triggers the release of the model drug inside the hair follicles.

Application of protease in a non-particulate form, e.g., a liquid formulation, as reported by Mak et al. [17], resulted in penetration of the protease only into the upper part of the hair follicles and, consequently, release of the model drug only occurred in this upper region. The present study enabled a deeper penetration also of the protease by being delivered via particles. It was demonstrated in the previous study that no significant decomposition of $\mathrm{P}_{\text {drug }}$ occurred when the particles interacted with $0.1 \%$ protease solution for 5 minutes [17]. An interaction time of one hour is required for the complete release of the model drug from $P_{\text {drug. }}$. It is understandable that the release of the model drug occurs only after the penetration process of the particles is almost completed, since penetration of the particle mixture into the hair follicles occurs mainly during the massage procedure. 
In the case of $P_{\text {drug }}$ being applied unaided onto skin, only a weak fluorescence signal from the model drug could be observed. A possible explanation for this effect could be the self-quenching between the fluorescence molecules, which might result from the higher concentration of the drug loaded in the particles. The results of the preliminary experiments demonstrated that the fluorescence intensity of the model drug was significantly higher when it had been released from the particles. This effect was also demonstrated in the present study, where a release of the model drug could be detected in the hair follicles even up to nearly $1 \mathrm{~mm}$ in depth.

Additionally, it was observed that, as a result of the interaction of both types of particles $\left(P_{\text {drug }}\right.$ and $\left.P_{\text {prot }}\right)$, the released model drug could be clearly detected in the sebaceous glands showing a strong fluorescence signal. This effect was not observed when the drug-loaded particles had been applied unaided. Consequently, it can be assumed that not the particles penetrate into the sebaceous glands, rather than exclusively the released model drug, which could be of interest for the development of new therapeutic strategies for the treatment of sebaceous gland associated diseases, such as acne vulgaris.

Under in vivo conditions, the drug being delivered close to the target structure must pass through the barrier of the hair follicles independently to reach the living cells and the blood circulation. The surfaces of the follicular openings are only initially keratinized while in the lower infundibulum, the corneocytes are smaller and crumbly and more penetrable, suggesting that the barrier is incomplete in this region [30, 31]. The efficiency of penetration of the released drug through the barrier of the hair follicles depends on the physicochemical properties and especially the size of the drug molecules [30]. Therefore, the penetration process of the released drug through the follicular barrier into the living cells must be separately evaluated for each single drug.

As far as safety aspects are concerned, the substances used in the present study can be applied in vivo onto human skin. In the present study, the substances had only a model character in order to demonstrate the perspective of triggering a drug release from the particles at the target structure inside the hair follicles. Therefore, no direct clinical application was intended in this work. 


\section{Conclusions}

Drug delivery by means of particles into hair follicles is a promising process, since the hair follicles offer several target sites of therapeutical interest. The cavities of the hair follicle permit long-term storage of drugs to achieve sustained release. The triggered release of drugs from the particles inside the hair follicles is essential in the delivery process. The results of the present study demonstrate an advanced triggering system based on a mixed particle formulation for the efficient release of the model drugs from the particle carrier inside hair follicles. The model drug was found to be distributed within the hair follicles, as well as in the sebaceous glands. Although $\underline{\text { the release system has still to be evaluated for further model drugs, drug molecules }}$ and particle types, it provides an important and promising new approach for the treatment of different types of skin diseases, for instance, acne vulgaris,

\section{Acknowledgements}

We would like to thank the Foundation "Skin Physiology" of the Donor Association for German Science and Humanities, and the German Academic Exchange Service (DAAD, G-HK027/10) for financial support. 
[1] M.C. Meinke, A. Patzelt, H. Richter, S. Schanzer, W. Sterry, A. Filbry, K. Bohnsack, F. Rippke, J. Galecka, R. Folster-Holst, J. Lademann, Prevention of follicular penetration: barrier-enhancing formulations against the penetration of pollen allergens into hair follicles, Skin Pharmacol Physiol, 24 (2011) 144-150.

[2] J.M. Moresi, T.D. Horn, Distribution of Langerhans cells in human hair follicle, J Cutan Pathol, 24 (1997) 636-640.

[3] M. Ohyama, Hair follicle bulge: a fascinating reservoir of epithelial stem cells, J Dermatol Sci, 46 (2007) 81-89.

[4] J. Lademann, F. Knorr, H. Richter, U. Blume-Peytavi, A. Vogt, C. Antoniou, W. Sterry, A. Patzelt, Hair follicles--an efficient storage and penetration pathway for topically applied substances. Summary of recent results obtained at the Center of Experimental and Applied Cutaneous Physiology, Charite -Universitatsmedizin Berlin, Germany, Skin Pharmacol Physiol, 21 (2008) 150-155.

[5] J. Lademann, H. Richter, S. Schanzer, F. Knorr, M. Meinke, W. Sterry, A. Patzelt, Penetration and storage of particles in human skin: perspectives and safety aspects, Eur J Pharm Biopharm, 77 (2011) 465-468.

[6] J. Lademann, H. Weigmann, C. Rickmeyer, H. Barthelmes, H. Schaefer, G. Mueller, W. Sterry, Penetration of titanium dioxide microparticles in a sunscreen formulation into the horny layer and the follicular orifice, Skin Pharmacol Appl Skin Physiol, 12 (1999) 247-256.

[7] R. Toll, U. Jacobi, H. Richter, J. Lademann, H. Schaefer, U. Blume-Peytavi, Penetration profile of microspheres in follicular targeting of terminal hair follicles, $\mathrm{J}$ Invest Dermatol, 123 (2004) 168-176.

[8] H. Schaefer, J. Lademann, The role of follicular penetration. A differential view, Skin Pharmacol Appl Skin Physiol, 14 Suppl 1 (2001) 23-27.

[9] A. Vogt, B. Combadiere, S. Hadam, K.M. Stieler, J. Lademann, H. Schaefer, B. Autran, W. Sterry, U. Blume-Peytavi, $40 \mathrm{~nm}$, but not 750 or 1,500 nm, nanoparticles enter epidermal CD1a+ cells after transcutaneous application on human skin, $\mathrm{J}$ Invest Dermatol, 126 (2006) 1316-1322.

[10] J. Lademann, H. Richter, A. Teichmann, N. Otberg, U. Blume-Peytavi, J. Luengo, B. Weiss, U.F. Schaefer, C.M. Lehr, R. Wepf, W. Sterry, Nanoparticles--an efficient carrier for drug delivery into the hair follicles, Eur J Pharm Biopharm, 66 (2007) 159-164.

[11] J. Lademann, H. Richter, U.F. Schaefer, U. Blume-Peytavi, A. Teichmann, N. Otberg, W. Sterry, Hair follicles - a long-term reservoir for drug delivery, Skin Pharmacol Physiol, 19 (2006) 232-236.

[12] J. Lademann, A. Patzelt, S. Schanzer, H. Richter, I. Gross, K.H. Menting, L. Frazier, W. Sterry, C. Antoniou, Decontamination of the skin with absorbing materials, Skin Pharmacol Physiol, 24 (2011) 87-92.

[13] J. Lademann, A. Patzelt, H. Richter, C. Antoniou, W. Sterry, F. Knorr, Determination of the cuticula thickness of human and porcine hairs and their potential influence on the penetration of nanoparticles into the hair follicles, J Biomed Opt, 14 (2009) 021014.

[14] A. Patzelt, H. Richter, F. Knorr, U. Schafer, C.M. Lehr, L. Dahne, W. Sterry, J. Lademann, Selective follicular targeting by modification of the particle sizes, J Control Release, 150 (2011) 45-48.

[15] D. Papakostas, F. Rancan, W. Sterry, U. Blume-Peytavi, A. Vogt, Nanoparticles in dermatology, Arch Dermatol Res, 303 (2011) 533-550. 
[16] M. Schneider, F. Stracke, S. Hansen, U.F. Schaefer, Nanoparticles and their interactions with the dermal barrier, Dermatoendocrinol, 1 (2009) 197-206.

[17] W.C. Mak, H. Richter, A. Patzelt, W. Sterry, K.K. Lai, R. Renneberg, J. Lademann, Drug delivery into the skin by degradable particles, Eur J Pharm Biopharm, 79 (2011) 23-27.

[18] M. Schafer-Korting, M. Holtje, H.C. Korting, H.D. Holtje, Innovative agents for actinic keratosis and nanocarriers enhancing skin penetration, Skin Pharmacol Physiol, 23 (2010) 6-14.

[19] X. Wu, K. Landfester, A. Musyanovych, R.H. Guy, Disposition of charged nanoparticles after their topical application to the skin, Skin Pharmacol Physiol, 23 (2010) 117-123.

[20] S. Biradar, P. Ravichandran, R. Gopikrishnan, V. Goornavar, J.C. Hall, V. Ramesh, S. Baluchamy, R.B. Jeffers, G.T. Ramesh, Calcium carbonate nanoparticles: synthesis, characterization and biocompatibility, J Nanosci Nanotechnol, 11 (2011) 6868-6874.

[21] W.C Mak, R. Georgieva, R., Renneberg, R.; Bäumler, H., Protein particles formed by protein activation and sponaneous sef-assembly, Advanced Functional Materials, 20 (2010) 4139-4144.

[22] U. Jacobi, R. Toll, H. Audring, W. Sterry, J. Lademann, The porcine snout--an in vitro model for human lips?, Exp Dermatol, 14 (2005) 96-102.

[23] W. Meyer, R. Schwarz, K. Neurand, The skin of domestic mammals as a model for the human skin, with special reference to the domestic pig, Curr Probl Dermatol, 7 (1978) 39-52.

[24] A. Patzelt, H. Richter, R. Buettemeyer, H.J. Huber, U. Blume-Peytavi, W. Sterry, J. Lademann, Differential stripping demonstrates a significant reduction of the hair follicle reservoir in vitro compared to in vivo, Eur J Pharm Biopharm, 70 (2008) 234238.

[25] J. Lademann, H. Richter, M. Meinke, W. Sterry, A. Patzelt, Which skin model is the most appropriate for the investigation of topically applied substances into the hair follicles?, Skin Pharmacol Physiol, 23 (2010) 47-52.

[26] N. Otberg, A. Patzelt, U. Rasulev, T. Hagemeister, M. Linscheid, R. Sinkgraven, W. Sterry, J. Lademann, The role of hair follicles in the percutaneous absorption of caffeine, Br J Clin Pharmacol, 65 (2008) 488-492.

[27] U. Blume-Peytavi, L. Massoudy, A. Patzelt, J. Lademann, E. Dietz, U. Rasulev, N. Garcia Bartels, Follicular and percutaneous penetration pathways of topically applied minoxidil foam, Eur J Pharm Biopharm, 76 (2010) 450-453.

[28] A. Teichmann, N. Otberg, U. Jacobi, W. Sterry, J. Lademann, Follicular penetration: development of a method to block the follicles selectively against the penetration of topically applied substances, Skin Pharmacol Physiol, 19 (2006) 216223.

[29] X. Liu, J.E. Grice, J. Lademann, N. Otberg, S. Trauer, A. Patzelt, M.S. Roberts, Hair follicles contribute significantly to penetration through human skin only at times soon after application as a solvent deposited solid in man, $\mathrm{Br} \mathrm{J}$ Clin Pharmacol, 72 (2011) 768-774.

[30] A.K. Patzelt, F.; Blume-Peytavi, U.; Sterry, W.; Lademann, J., Hair follicles, their disorders and their opportunities, Drug Discovery Today: Disease Mechanisms, 5 (2008) 173-181.

[31] G.K. Menon, A.M. Kligman, Barrier functions of human skin: a holistic view, Skin Pharmacol Physiol, 22 (2009) 178-189.

[XX] S. Biradar, P. Ravichandran, R. Gopikrishnan, V. Goornavar, J.C. Hall, V. Ramesh, S. Baluchamy, R.B. Jeffers, G.T. Ramesh, Calcium carbonate 
nanoparticles: synthesis, characterization and biocompatibility, Journal of Nanoscience and nanotechnology 11 (2011) 6868-6874. 


\section{Figure captions}

Figure 1. Schematic diagram of penetration and release concept

Figure 2. SEM images showing the surface morphologies of the $P_{\text {drug }}(a)$ and $P_{\text {prot }}$ particles (b). The top inset shows the enlarged image.

Figure 3. Differences in the fluorescence intensity of the model drug FITC when $P_{\text {drug }}$ and $P_{\text {prot }}$ were applied in a mixture (a) or when $P_{\text {drug }}$, was applied unaided without protease particles (b)

Figure 4. Average values of the penetration depths of the model drug FITC and the rhodamine-labelled protease particles in $\mu \mathrm{m}$

Figure 5. Distribution of the model drug FITC in the hair follicle and the sebaceous gland when $P_{\text {drug }}$ and $P_{\text {prot }}$ were applied as a mixture (a) or when $P_{\text {drug }}$ was applied unaided without protease particles (b) 